\title{
Imaging of coherent fields through lenslike systems
}

\author{
Amnon Yariv \\ California Institute of Technology, Pasadena, California 91125
}

Received June 8, 1994

I derive the imaging condition for the complex amplitude of a monochromatic field by a sequence of lenslike elements.

In this Letter I consider the problem of imaging a coherent electromagnetic field by a general axisymmetric lenslike system, as illustrated in Fig. 1. The individual elements that compose the system are each describable by an $A B C D$ ray matrix, and the propagation between the input plane $\left(x_{0}, y_{0}\right)$ and the output plane $\left(x_{1}, y_{1}\right)$ is thus governed by the overall system matrix:

$$
\left|\begin{array}{cc}
A & B \\
C & D
\end{array}\right|=\left|\begin{array}{ll}
A_{n} & B_{n} \\
C_{n} & D_{n}
\end{array}\right| \cdots\left|\begin{array}{cc}
A_{1} & B_{1} \\
C_{1} & D_{1}
\end{array}\right|
$$

In an important extension of the eikonal formalism, Baues $^{1}$ and Collins ${ }^{2}$ showed that the output and input complex fields in our problem are related by
In the case of a lossless system, $A D-B C=1$, so that Eq. (5) can be written as

$$
\begin{aligned}
f_{1}\left(x_{1} y_{1}\right)= & \frac{\exp (-i k L)}{A} f_{0}\left(\frac{x_{1}}{A}, \frac{y_{1}}{A}\right) \\
& \times \exp \left[-i \frac{k C}{2 A}\left(x_{1}{ }^{2}+y_{1}{ }^{2}\right)\right] .
\end{aligned}
$$

Equation (6) is the central result of this Letter. It shows that when $B=0$ the output is an exact, scaled replica of the input field except for a quadratic phase factor. ${ }^{3,4}$ The image magnification is $A$. The generalized imaging condition is thus $B=0.5,6$

As an example, I apply the formalism to the simple case of imaging by a single thin lens, as illustrated in Fig. 2. In this case,

$f_{\mathrm{i}}\left(x_{1}, y_{1}\right)=\frac{i k}{2 \pi B} \exp (-i k L) \iint_{\Sigma_{0}} f_{0}\left(x_{0}, y_{0}\right) \exp \left\{\left(-\frac{i k}{2 B}\right)\left[D\left(x_{1}{ }^{2}+{y_{1}}^{2}\right)-2 x_{1} x_{0}-2 y_{1} y_{0}+A\left(x_{0}{ }^{2}+y_{0}{ }^{2}\right)\right]\right\} \mathrm{d} x_{0} \mathrm{~d} y_{0}$.

By rearranging and manipulating the exponent we can rewrite Eq. (2) as

$$
\begin{aligned}
f_{1}\left(x_{1}, y_{1}\right)= & \frac{i k}{2 \pi B} \exp \left[\left(-\frac{i k}{2 B}\right)\left(D-\frac{1}{A}\right)\left(x_{1}{ }^{2}+y_{1}^{2}\right)\right] \\
& \times \iint_{\Sigma_{0}} f_{0}\left(x_{0}, y_{0}\right) \exp \left\{\left(-\frac{i k}{2 B}\right)\right. \\
& \left.\times\left[A\left(x_{0}-\frac{x_{1}}{A}\right)^{2}+A\left(y_{0}-\frac{y_{1}}{A}\right)^{2}\right]\right\} \mathrm{d} x_{0} \mathrm{~d} y_{0} .
\end{aligned}
$$

Here I consider the function $\exp \left[-(i k A / 2 B)\left(x_{0}-\right.\right.$ $\left.\left.x_{1} / A\right)^{2}\right]$.

I will show formally, at the end of this Letter, that

$$
Y(x) \equiv \lim _{B \rightarrow 0} \sqrt{\frac{i}{2 \pi B}} \exp \left(-i \frac{x^{2}}{2 B}\right)=\delta(x)
$$

where $\delta(x)$ is the Dirac delta function, so that in the limit $B \rightarrow 0$

$$
\begin{aligned}
f_{1}\left(x_{1}, y_{1}\right)= & \frac{\exp (-i k L)}{A \rightarrow 0} f_{0}\left(\frac{x_{1}}{A}, \frac{y_{1}}{A}\right) \\
& \times \exp \left[-i \frac{k(D A-1)}{2 A B}\left(x_{1}^{2}+y_{1}^{2}\right)\right] .
\end{aligned}
$$

$$
\begin{aligned}
\left|\begin{array}{ll}
A & B \\
C & D
\end{array}\right| & =\left|\begin{array}{cc}
1 & d_{i} \\
0 & 1
\end{array}\right|\left|\begin{array}{cc}
1 & 0 \\
-\frac{1}{f} & 1
\end{array}\right|\left|\begin{array}{cc}
1 & d_{0} \\
0 & 1
\end{array}\right| \\
& =\left|\begin{array}{cc}
1-\frac{d_{i}}{f} & d_{0} d_{i}\left(\frac{1}{d_{0}}+\frac{1}{d_{i}}-\frac{1}{f}\right) \\
-\frac{1}{f} & 1-\frac{d_{0}}{f}
\end{array}\right| .
\end{aligned}
$$

The imaging condition $B=0$ in this case assumes the familiar form of geometrical optics,

$$
\frac{1}{f}=\frac{1}{d_{0}}+\frac{1}{d_{i}}
$$

from which it follows directly that $A=1-d_{i} / f=$ $-d_{i} / d_{0}=-M$, where $M \equiv d_{i} / d_{0}$ is the magnification factor. In a similar fashion I show that $C / A=M / f$ and $D=-M^{-1}$, so that

$$
\begin{aligned}
f_{1}(x, y)= & -\frac{1}{M} \exp \left\{-i\left[k L+\frac{k\left(x^{2}+y^{2}\right)}{2 M f}\right]\right\} \\
& \times f_{0}\left(-\frac{x}{M},-\frac{y}{M}\right) .
\end{aligned}
$$

This special case reduces to a result given by Goodman. ${ }^{7}$ Another special case of imaging by a quadratic index fiber is considered in Ref. 5 . I have thus derived the imaging relation for a coherent electromagnetic field by a system of lenslike elements. 


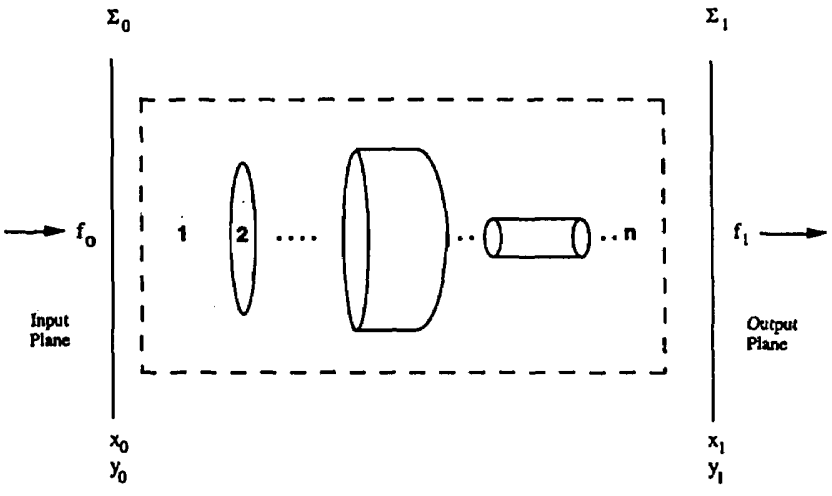

Fig. 1. Generalized lenslike system.

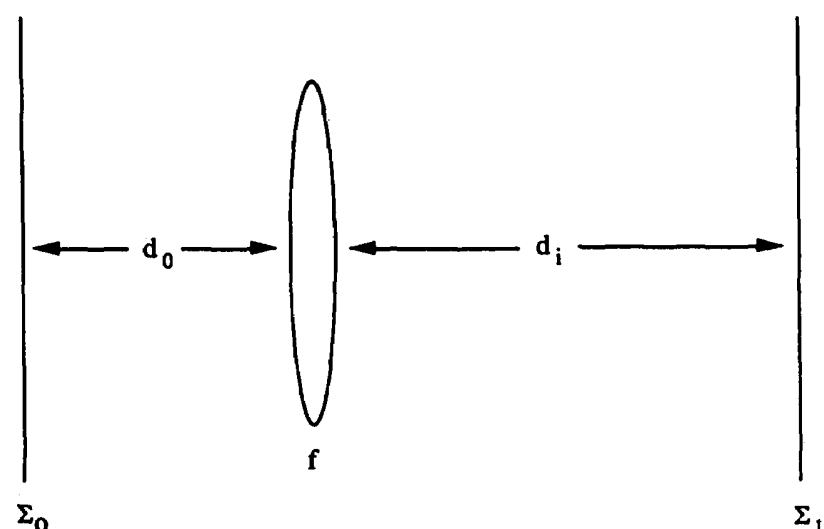

Fig. 2. Imaging by a thin lens.

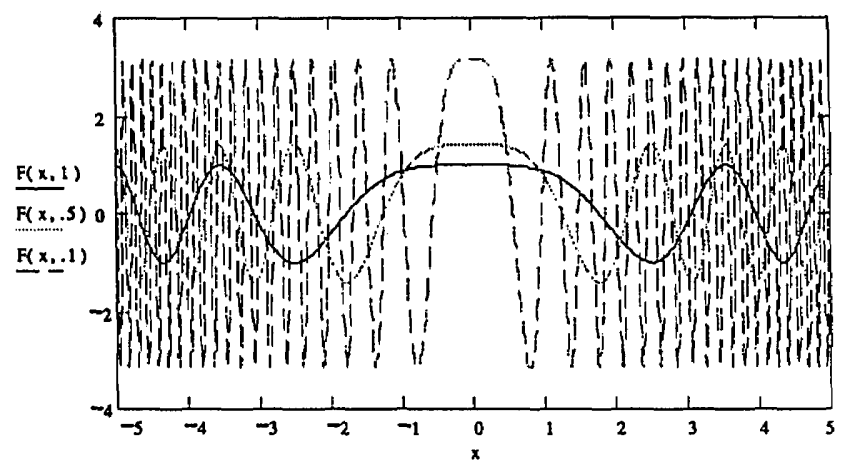

Fig. 3. Function $F(x, B)=(2 \pi B)^{-1 / 2} \cos \left(x^{2} / 2 B\right)$ for $B=1,0.5,0.1$.

To prove, as claimed above, that

$$
Y(t) \equiv \lim _{B \rightarrow 0}\left(\frac{i}{2 \pi B}\right)^{1 / 2} \exp \left(-i \frac{2 t^{2}}{2 B}\right)=\delta(t),
$$

we need to show that

$$
\int_{-\infty}^{\infty} Y(t) \mathrm{d} t=1
$$

and that

$$
\int_{t_{1}}^{t_{2}} Y(t) \mathrm{d} t=0
$$

for any interval $t_{1} \ldots t_{2}$ that does not contain the origin. To appreciate qualitatively the nature of the function $(i / 2 \pi B)^{1 / 2} \exp \left(-i x^{2} / 2 B\right)$, consider its projection,

$$
F\left(x, B \equiv\left(\frac{1}{2 \pi B}\right)^{1 / 2} \cos \left(\frac{x^{2}}{2 B}\right)\right.
$$

Figure 3 shows plots of $F(x, B)$ for $B=1,0.5,0.1$. Equation (11) reflects the fact that the main contribution to the integral is from the first few oscillations near the origin (the area under a given number of peaks is independent of $B$ ), whereas Eq. (12) follows from the fact that, when $B \rightarrow 0, t_{1}$ and $t_{2}$ in the normalized variable $x=t / \sqrt{B}$ of Fig. 3 tend to infinity. More rigorously, I employ the definition of the Fresnel cosine and sine integrals ${ }^{8} C(\omega)$ and $S(\omega)$, respectively,

$$
\begin{aligned}
& C(\omega)=\int_{0}^{\omega} \cos \left(\frac{\pi}{2} \tau^{2}\right) \mathrm{d} \tau, \\
& S(\omega)=\int_{0}^{\omega} \sin \left(\frac{\pi}{2} \tau^{2}\right) \mathrm{d} \tau, \\
& C(\infty)=S(\infty)=0.5
\end{aligned}
$$

to express Eq. (11) as

$$
\begin{aligned}
\int_{t_{1}}^{t_{2}} Y(\mathrm{t}) \mathrm{d} t= & \frac{1}{2}(1+i) \lim _{B \rightarrow 0}\left\{C\left(\frac{t_{2}}{\sqrt{\pi B}}\right)-C\left(\frac{t_{1}}{\sqrt{\pi B}}\right)\right. \\
& \left.-i\left[S\left(\frac{t_{2}}{\sqrt{\pi B}}\right)-S\left(\frac{t_{1}}{\sqrt{\pi B}}\right)\right]\right\} \\
= & \frac{1}{2}(1+i)\{C(\infty)-C(\infty) \\
& -i[S(\infty)-S(\infty)]\}=0
\end{aligned}
$$

when $t_{1}$ and $t_{2}$ have the same sign.

In a similar fashion I show that

$$
\int_{-\infty}^{\infty} Y(t) \mathrm{d} t=(1+i)[C(\infty)-i S(\infty)]=1
$$

The author is indebted to J. Franklin for useful mathematical discussion. This research was supported by the U.S. Army Research Office, Durham, North Carolina.

\section{References}

1. P. Baues, Optoelectronics 1, 37 (1969).

2. S. B. Collins, J. Opt. Soc. Am. 60, 1168 (1970),

3. G. N. Lawrence and S. W. Hwang, Appl. Opt. 31,5201 (1992).

4. H. T. Yura and S. G. Hanson, J. Opt. Soc. Am. A 4, 1931 (1987).

5. The identification of the condition $B=0$ with the imaging condition of a lenslike system is given in A. Yariv, J. Opt. Soc. Am. 66, 301 (1976) as well as in Ref. 6.

6. A. E. Siegman, Lasers (University Science, Mill Valley, Calif., 1986), p. 596.

7. J. W. Goodman, Introduction to Fourier Optics (McGraw-Hill, New York, 1968), p. 96.

8. M. Born and Wolf, Principles of Optics, 5th ed. (Pergamon, Oxford, 1975), p. 430. 